\title{
Natural Radiation Events in CCD Imagers at Ground Level
}

\author{
T. Saad Saoud, S. Moindjie, D. Munteanu, J.L. Autran ${ }^{*}$ \\ Aix Marseille Université, CNRS, Université de Toulon, IM2NP UMR 7334, 13397 Marseille, France
}

\begin{abstract}
In Charged Coupled Devices (CCDs), radiation-induced events generate electron hole pairs in silicon that cause artifacts and contribute to degrade image quality. In this work, the impact of natural radiation at ground level has been characterized at sea level, in altitude and underground for a commercial full-frame CCD device. Results have been carefully analyzed in terms of event shape, size and hourly rates. The respective contributions of atmospheric radiation and telluric contamination from ultra-traces of alpha-particle emitters have been successfully separated and quantified. Experimental results have been compared with simulation results obtained from a dedicated radiation transport and interaction code.
\end{abstract}

\section{Keywords}

Charge-Coupled Devices (CCD), terrestrial cosmic rays, atmospheric neutrons, protons, muons, alpha-particle emitters, Monte Carlo simulation, underground test, altitude test.

\section{Highlights}

- Image artifacts induced by natural radiation in CCD images are investigated

- Radiation-induced events are analyzed in terms of pixel size and hourly rates

- Numerical simulations give the contributions of the different particles to the event rates

\section{* Corresponding author:}

Prof. Jean-Luc Autran

IM2NP - UMR CNRS 7334

Faculté des Sciences - Service 142

Avenue Escadrille Normandie

F-13397 Marseille Cedex 20 France

Phone: + 33 (0)413 594627

Fax: +33(0)491288531

Email: jean-luc.autran@univ-amu.fr 


\section{Introduction}

As most solid-state devices, Charged Coupled Devices (CCDs) are known to be very sensitive to natural or artificial sources of radiation [1-2]. Depending on the context of use of such devices, this extreme sensitivity is an advantage if one tries to detect radiation [3-17] or a disadvantage if we try to avoid them [18]. Indeed, the interactions of single ionizing particles with the CCD materials generate electron-hole pairs that can be partially or totally collected at silicon (i.e. pixel) level, resulting in image artifacts. The effects are not permanent and the spurious charge is swept out during readout, but these single event effects (SEE) constitute a significant source of noise in the image/video data. Named "cosmic rays" by astronomers (because mainly induced by secondary cosmic rays at terrestrial level) [19], these nonsense signals directly affect the reliability of high performance CCD imagers used in astronomy for example [20].

Schematically, SEEs in CCDs can be generated by direct or indirect ionization [21], as summarized and illustrated in Fig. 1. On one hand, direct ionization (Fig. 1 left) is achieved through Coulomb interactions between a charged particle and atoms of the device. The charged particle strips electrons of atoms as it passes through the device thereby causing ionizations. Heavy ions (including charged nuclear recoils), low energy protons and muons directly ionize matter. As a function of the incidence angle of the particle with respect to the CCD plan, different signatures (spots, straight lines) can be obtained [22]. On the other hand, indirect ionization (Fig. 1 right) is of concern for atmospheric neutrons and high-energy protons $(>100 \mathrm{MeV})$ that are able to ionize by collision with the target nuclei. Neutrons ionize indirectly, they do not interact via the Coulomb force, and so they can travel through several centimeters of material without interacting with other particles and can remain undetected with CCD. Indirect ionization is accomplished through two mechanisms: elastic and inelastic scattering [23]. Elastic scattering occurs when a neutron knocks out a target nucleus from its lattice but the nucleus remains in the same energy state. During inelastic scattering, the striking neutron interacts with the target nucleus such that the nucleus captures the neutron and thereby the nucleus becomes an isotope. The isotope then deexcites by the emission of secondary charged and uncharged radiation. The residual nucleus and the evaporation products may be highly ionizing and are able to deposit significant amounts of charge at various locations in a device and cause SEEs. Fig. 1 (right) illustrates the case of an indirect ionization event induced by a neutron-silicon interaction within the volume of the 
CCD. As a function of the number and the momentum direction of the reaction products, the signature of the event can be more complex than a simple spot or straight line.

In this work, we used a commercial CCD-based astronomical camera to precisely detect, count and characterize (in terms of pixel size, hourly rate and related charge event) artifacts induced by natural radiation at ground level from the continuous analysis of images captured in total darkness. In order to also isolate and characterize events directly induced by the internal and residual radioactivity of the CCD materials, we performed experiments deeply underground to suppress atmospheric radiation. Three different locations have thus been considered: at sea level, underground and at mountain altitude. Measurements reported in this work correspond to long-term experiments, with typical durations of several months.

Section 2 of the present paper details the experimental setup, the acquisition procedure and the image processing for radiation-induced event characterization. Experimental results for the different locations are reported and discussed in Section 3. Finally, in Section 4, these data have been compared with results deduced from numerical simulation performed with a dedicated radiation transport and interaction code developed in the framework of this study.

\section{Experimental details}

The different experiments of this work have been conducted using a USB2.0 CCD monochrome camera (model Atik 383L [24], see Fig. 1) taking one full image per minute (i.e. 1 frame/min) in the complete darkness. The KAF-8300 image sensor [25] is a high performance monochrome full frame CCD with a square pixel $\left(5.4 \times 5.4 \mu \mathrm{m}^{2}\right)$ array and $8.6 \times 10^{6}$ effective pixels $\left(2.428 \mathrm{~cm}^{2}\right.$ of active surface $)$. Each pixel contains a lateral overflow drain for blooming protection (only in case of high light level conditions). The camera is based on a 16bit Analog to Digital Converter (ADC) and is thermally stabilized. The system contains a cooled thermoelectric device that maintains the CCD approximately $40^{\circ} \mathrm{C}$ below the room temperature, reducing by several orders of magnitude the dark noise due to thermal generation [24]. The main characteristics of the image sensor are summarized in the table of Fig. 1.

In addition to the camera, the experimental setup (Fig. 1) also includes a Windows 7 computer and a homemade image processing software integrating Matlab routines. The program performs image cleaning and analysis, radiation-induced event extraction and the storage of these "event images" and related information into a MySQL open source database 
server running on the same PC. The database also contains information about all CCD artifacts, i.e. damaged and instable pixels, subjected to random telegraph signal noise or other characteristic electrical instabilities [4-6]. These additional data are used by the program to eliminate all image instabilities not directly linked with radiation events.

At the end of each acquisition period ( 1 minute) in the complete darkness, the CCD is read and a raw image is returned to the computer. This image is cleaned (noise removal) by subtracting a reference dark frame (obtained from a combination of multiple dark frames at the same temperature to get a better model for the noise in an image). Radiation-induced events are then detected by applying to this new image a series of mathematical treatments that isolate pixels or group(s) of connected pixels with an electrical charge clearly above the background and verifying certain threshold criteria. This operation consists in identifying pixels above a first threshold value and to examine if the neighboring pixels (up to the second neighbors) are also above a second value (inferior to the first threshold but superior to the image background).

Fig. 2 shows a panel of typical events detected during a one-year experiment performed at sea level. The deposited charge corresponding to each detected event is estimated by summing the values (i.e. the readings) of the event pixels, considering a linear dependence between a pixel value and its electrical charge up to the charge saturation. Similarly to [17], the detected events are automatically classified by the software into single pixel event (SPE) that corresponds to isolated pixels or into multiple pixel event (MPE) that corresponds to a group of adjacent or neighboring pixels (i.e. having pixel connectivity). Finally, the software estimated the corresponding hourly event rates (single pixel rate, SPER, and multiple pixel rate, MPER) occurring during the experiment.

\section{Experimental results}

Experimental campaigns of measurements were performed in three different locations with exactly the same camera and the same setup: i) at sea level in Marseille [26] during more than one year (14 months), ii) underground at the underground laboratory of Modane (LSM [27], under $1700 \mathrm{~m}$ of rock, equivalent to $4800 \mathrm{~m}$ under water) during two weeks and iii) at mountain altitude $(2552 \mathrm{~m})$ on the ASTEP platform [28] during three months. Underground measurements represent the only way to screen the atmospheric radiation and to directly measure, i.e. quantify, the occurrence of events due to the residual ultra-traces of alpha 
radioactivity present in the CCD materials and packaging (in particular in the quartz optical window located in front of the CCD). On the contrary, altitude measurements are chosen to increase the atmospheric radiation by a factor corresponding to the acceleration factor (AF) of the considered site. AF is defined by the relative particle flux (total integral flux above a certain energy, generally $1 \mathrm{MeV}$ ) at the test location with respect to the reference value of the same quantity evaluated at sea level (New York City) [23]. For ASTEP, AF $\approx 6$ [29] for the atmospheric neutron flux integrated above $1 \mathrm{MeV}$; this value is higher for atmospheric protons $(\mathrm{AF} \approx 8)$ and lower for muons $(\mathrm{AF} \approx 2)$ since the dependence with altitude of the integrated flux is different for these particles [30].

Fig. 3 reports the experimental values of the event hourly rate detected during the three experiments. These values correspond to a global event rate, i.e. representing the summation of both MPE and SPE event rates. Altitude event rate is only $2.4 \times$ the value measured at sea level. By comparing the values obtained at LSM and in Marseille, we can deduce that more than the half $(58 \%)$ of detected events at sea level is in fact due to the CCD chip internal alpha radioactivity. This contribution of chip radioactivity decreases to $27 \%$ for altitude measurements, mainly dominated by atmospheric radiation in this case. Subtracting the event rate detected underground $\left(2.72 \mathrm{~h}^{-1}\right)$ to the values measured at sea level and in altitude gives the contributions of the sole atmospheric radiation: we obtain $1.98 \mathrm{~h}^{-1}$ for Marseille and 8.48 $\mathrm{h}^{-1}$ for ASTEP. In this case, these values show that the atmospheric event rate increases by a factor $\approx 4.3$ for ASTEP with respect to sea level, larger than the factor 2.4 previously deduced from raw measurements taking into account the chip radioactivity contribution. In order to understand the exact contributions of the different atmospheric particle types to the observed event rates, numerical simulation have been carried out (see the next section).

\section{Modeling and numerical simulation}

The complete modeling and numerical simulation of the different experiments has been performed following a methodology successfully experimented in a previous work concerning another type of CCD sensor and camera [17]. From device performances specifications [25] combined with a chip analysis conducted on a sacrificed device with techniques and instruments classically used in IC failure analysis, we constructed a simplified but realistic 3D model of the complete pixel array considering the extracted dimensions, material and layer composition and thicknesses. The geometry has been modeled using a $\mathrm{C}++$ computational 
geometry library developed at IM2NP. The EXPACS atmospheric radiation model [31] has been also implemented in the code to compute the energy distributions of neutrons, protons and muons fluxes precisely corresponding to sea level and altitude test locations at the measurement periods. In addition, for alpha-particle emitters, both ${ }^{238} \mathrm{U}$ and ${ }^{232} \mathrm{Th}$ decay chains [23] have been simulated for contaminants uniformly distributed (for simplicity) in the bulk of all chip material layers. The interactions of neutrons and high-energy protons with circuit materials have been implemented on the basis of pre-calculated Geant4 databases [32]. For all other ionizing particles (secondary products, low energy muons and protons, alpha particles), a direct ionization process has been considered based on a numerical modeling of the SRIM tables [33-34]. Values for muons have been estimated by applying mass scaling to proton transport tables, as specified in [35]. When a particle interacts with the CCD materials, the code evaluates the amount of charge (energy) directly deposited into the pixels and the additional amount of charge collected by the pixels from the substrate, considering a diffusion-collection model in the silicon region described in [17]. For each interaction event impacting the pixel array, the code calculates the resulting numerical image and save it for post-processing and comparison with experimental signatures.

Extensive simulations considering 500 millions of atmospheric neutrons, 10 millions of protons and 1 million of muons, generated from the EXPACS model for the considered

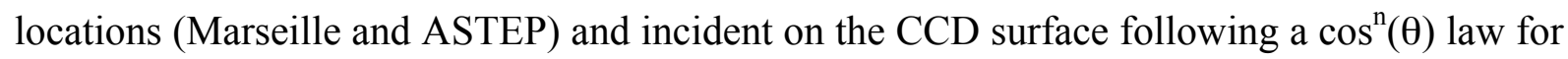
the zenithal angle $\theta(n=3.5$ for neutrons and $n=2$ for protons and muons [34]), have been performed. In addition, 1 million of alpha particles, emitted from a random location in the CCD materials with characteristics derived from the ${ }^{238} \mathrm{U}$ and ${ }^{232} \mathrm{Th}$ decay chains, have been also considered. The relatively high experimental pixel event rate measured underground $\left(2.72 \mathrm{~h}^{-1}\right)$ has been numerically reproduced by forcing the uranium (and thorium) concentration to the value of $1.9 \mathrm{ppm}$ in the device. This value is lower than the value extracted in [33] for another imager and camera, but it also reflects a residual radioactivity problem in the chip and/or in the packaging. As reported in [33], the quartz cover glass (optical window), just in front of the sensor, should be at the origin of this problem since such a contamination with alpha radioactive impurities has been already observed in such materials [37-38]. Other possible origins of such a radioactive contamination could be linked to the antireflective coatings [24] present on both sides of the optical glass or to the chip bonding 
materials. This point should require further investigations since it appears to be recurrent on different CCD and CMOS sensors [33, 37, 38].

From the different simulation batches performed for all particle types, quantitative results presented in Figs. 4 and 5 have been extracted. Fig. 4 shows that the global event rates obtained by simulation, i.e. the sum of the pixel event rates for both atmospheric (neutrons + protons + muons and telluric contributions) match very well the experimental values at ground level. In more details, these results show that neutrons, muons and protons have very different weights in the total event rate observed at sea level or at mountain altitude: neutrons weakly interact with the CCD and are responsible of only a few percents of the detected events (respectively $7 \%$ and $17 \%$ of the atmospheric radiation-induced events observed at sea level an in altitude) whereas muons are at the origin of the majority of events (respectively $84 \%$ and $62 \%$ of the "atmospheric" events at sea level an in altitude). Finally, proton contribution is found negligible at sea level (9\%) but more significant in altitude (21\%), due to an important increase of the amplification factor at ASTEP altitude (with respect to sea level) previously discussed (see Section 3). These new results confirm and show the importance of muon and proton contributions in the CCD response when directly exposed to atmospheric radiation at ground level, as previously highlighted in [16-17]. To conclude, Fig. 5 shows the comparison between the experimental and the simulated distributions of event sizes expressed in number of pixels. The experimental distribution corresponds to 14 months of measurement at sea level. Simulations are found to globally well reproduce the experiment distribution even if these calculations slightly underestimate the occurrence of small events (up to 3 pixels in size) and overestimate larger events ( $>4$ pixels). Differences observed specially for large events ( $>6$ pixels) could be attributed to the finite duration of the measurements (one year), certainly not sufficient to reach a correct statistics for the largest (and thus the rarest) events. Finally, note that the cumulated effect of all the simplifications considered in the present simulation approach (CCD geometry, charge transport model, electrical pixel response, etc.) could also partially explain the differences observed between the two distributions of Fig. 5.

\section{Conclusion}

In conclusion, in this work we used a commercial CCD-based astronomical camera to detect and characterize the artifacts induced by natural radiation at ground level from the continuous 
analysis of images (acquired in the complete darkness one time per minute) at three different locations: at sea level, underground and at mountain altitude. Measurements reported in this work thus correspond to real-time long-term experiments, with typical durations of several months. Experimental data have been carefully analyzed in terms of event size and hourly rates and compared with simulation results obtained from a dedicated radiation transport and interaction code adapted to the current CCD geometry. The ensemble provides a set of experimental and simulation results quantifying the importance of such transient instabilities in CCD operation. In addition, the study gives us the opportunity to improve our modeling approach and to optimize our simulation code. The comparison between sea level and underground measured data indicated that more than half of the detected events at sea level is due to the CCD chip internal alpha radioactivity, a recurrent problem previously observed on various devices. In altitude measurements, the contribution of the chip radioactivity reduces to $27 \%$ in the CCD response, because the atmospheric radiation dominates in this case. The occurrence of the atmospheric radiation-induced events is found to increase by a factor $3 \times$ for altitude measurements with respect to sea level experiments. Numerical simulation has been finally performed in order to understand the exact contribution of the different atmospheric particle types, evidencing the predominant role of muons (and secondarily protons) in the response of the CCD device at ground level.

\section{Acknowledgments}

The authors would like to acknowledge Michel Zampaolo, Guillaume Warot, Fabrice Piquemal (LSM, CEA-CNRS, Modane, France), Bertrand Gautier and Cécile Di Léone (IRAM Observatory, Dévoluy, France) for their technical support during the underground and altitude experiments, respectively performed at LSM and on the ASTEP platform hosted by IRAM.

\section{References}

[1] D. Durini (Editor), High Performance Silicon Imaging: Fundamentals and Applications of CMOS and CCD sensors, Woodhead Publishing Series in Electronic and Optical Materials, Elsevier, 2014.

[2] J. Ohta, Smart CMOS Image Sensors and Applications, CRC Press, 2007. 
[3] T. S. Lomheim, R.M. Shima, J. R. Angione,W. F.Woodward, D. J. Asman, R. A. Keller, and L. W. Schumann, "Imaging charge-coupled device (CCD) transient response to 17 and $50 \mathrm{MeV}$ proton and heavy-ion irradiation”, IEEE Trans. Nucl. Sci., vol. 37, no. 6, pp. 1876-1885, Dec. 1990.

[4] R. Bailey, C. J. S. Damerell, R. L. English, A. R. Gillman, A. L. Lintern, S. J. Watts, and F. J. Wickens, "First measurements of efficiency and precision of CCD detectors for high energy physics”, Nuclear Instruments and Methods in Physics Research, vol. 213, no. 2-3, pp. 201-215, Aug. 1983.

[5] G. R. Hopkinson, "Radiation effects on solid state imaging devices", Radiation Physics and Chemistry, vol. 43, no. 1/2, pp. 79-91, 1994.

[6] G. R. Hopkinson, C. J. Dale, and P. W. Marshall, "Proton effects in charge-coupled devices”, IEEE Trans. Nucl. Sci., vol. 43, no. 2, pp. 614-627, Apr. 1996.

[7] A.M. Chugg, R. Jones, P. Jones, P. Nieminen, A. Mohammadzadeh, M. S. Robbins, and K. Lovell, “CCD miniature radiation monitor”, IEEE Trans. Nucl. Sci., Vol. 49, pp. 1327-1332, 2002.

[8] A.M. Chugg, R. Jones, M.J. Moutrie, C.S. Dyer, K.A. Ryden, P.R. Truscott, J.R. Armstrong, D.B.S. King, “Analyses of CCD images of nucleon-silicon interaction events”, IEEE Trans. Nucl. Sci., Vol. 51, pp. 2851-2856, 2004.

[9] Z. Török and S. P. Platt, “Application of imaging systems to characterization of single-event effects in high-energy neutron environments”, IEEE Trans. Nucl. Sci., Vol. 53, pp. 3718-3725, 2006.

[10] S.P. Platt and Z. Török, "Analysis of SEE-inducing charge generation in the neutron beam at The Svedberg Laboratory”, IEEE Trans. Nucl. Sci., Vol. 54, pp. 1163-1169, 2007.

[11] A.M. Chugg, A.J. Burnell, and R. Jones, "Webcam observations of SEE events at the Jungfraujoch research station", in Proc. $9^{\text {th }}$ European Conference on Radiation Effects on Components and Systems (RADECS 2007), paper PD-1, 2007.

[12] S.P. Platt, B. Cassels, and Z. Török, "Development and application of a neutron sensor for single event effects analysis”, J. Phys.: Conf. Ser., vol. 15, pp. 172-176, 2005.

[13] X.X. Cai, S.P. Platt, W. Chen, "Modelling Neutron Interactions in the Imaging SEE Monitor”, IEEE Trans. Nucl. Sci., Vol. 56, pp. 2035-2041, 2009. 
[14] X.X. Cai and S. P. Platt, "Modeling Neutron Interactions and Charge Collection in the Imaging Single-Event Effects Monitor", IEEE Trans. Nucl. Sci., Vol. 58, pp. 910-915, 2011.

[15] X.X. Cai, S.P. Platt, S.D. Monk, "Design of a Detector for Characterizing Neutron Fields for Single-Event Effects Testing", IEEE Trans. Nucl. Sci., Vol. 58, pp. 11231128, 2011.

[16] G. Hubert, A. Cheminet, T. Nuns, and V. Lacoste, “Atmospheric Radiation Environment Analyses Based-on CCD Camera, Neutron Spectrometer and MultiPhysics Modeling”, IEEE Trans. Nucl. Sci., Vol. 60, p. 4660-4667, 2013.

[17] T. Saad Saoud, S. Moindjie, J.L. Autran, D. Munteanu, F. Wrobel, F. Saigné, P. Cocquerez, L. Dilillo, M. Glorieux, "Use of CCD to Detect Terrestrial Cosmic Rays at Ground Level: Altitude vs. Underground Experiments, Modeling and Numerical Monte Carlo Simulation”, IEEE Trans. Nucl. Sci., Vol. 61, pp. 3380-3388, 2014.

[18] A.R. Smith et al. "Radiation events in astronomical CCD images". Proc. SPIE 4669, Sensors and Camera Systems for Scientific, Industrial, and Digital Photography Applications III, pp. 172-183, 2002.

[19] S.B. Howell, Handbook of CCD Astronomy, Cambridge University Press, 2006.

[20] D. Groom, “(Unwanted) radiation events in astronomical CCD images”. SPIE02 Rad Events in CCD's, 2002. Online: http://snap.lbl.gov/ccdweb/ccdrad talk spie02.pdf

[21] D. Munteanu and J.L. Autran, "Modeling and Simulation of Single-Event Effects in Digital Devices and ICs”, IEEE Trans. Nucl. Sci., Vol. 55, pp. 1854-1878, 2008.

[22] Z. Török, "Development of image processing systems for cosmic ray effect analysis", Ph.D. Thesis, University of Central Lancashire (UK), 2007.

[23] J.L. Autran and D. Munteanu, Soft Errors: from particles to circuits, Taylor \& Francis/CRC Press, 439 p., 2015.

[24] ATIK 383L camera. Online : http://3ainmfntxe31vi9qd1pxgpd1.wpengine.netdnacdn.com/wp-content/uploads/2015/07/Atik383L-Manual.pdf

[25] Kodak KAF-8300 image sensor performance specification, revision 5.4 MTD/PS0996, 2010. Online:

http://www.kodak.com/ek/uploadedFiles/Content/Small_Business/Images_Sensor_Sol utions/Datasheets(pdfs)/KAF-8300LongSpec.pdf 
[26] IM2NP Laboratory, Avenue Escadrille Normandie Niemen, F-13397 Marseille Cedex 20, France. GPS coordinates: 43.337838, 5.412063.

[27] Underground Laboratory of Modane (LSM, CEA-CNRS). Located along the Frejus road tunnel in Savoy. Online: http://www-lsm.in2p3.fr

[28] J.L. Autran, S. Serre, D. Munteanu, S. Martinie, S. Semikh, S. Sauze, S. Uznanski, G. Gasiot, P. Roche, "Real-Time Soft-Error Testing of 40nm SRAMs", International Reliability Physics Symposium (IRPS'2012), Anaheim, USA, April 15-19, 3C-5, 2012.

[29] J.L. Autran, D. Munteanu, S. Moindjie, T. Saad Saoud, S. Sauze, G. Gasiot, P. Roche, “ASTEP (2005-2015): Ten Years of Soft Error and Atmospheric Radiation Characterization on the Plateau de Bure," Microelectronics Reliability, Vol. 55, pp. 1506-1511, 2015.

[30] J.L. Autran, D. Munteanu, "Radiation Effects and COTS at Ground Level", Microelectronics Reliability, Vol. 55, pp. 2147-2153, 2015.

[31] T. Sato, “Analytical Model for Estimating Terrestrial Cosmic Ray Fluxes Nearly Anytime and Anywhere in the World: Extension of PARMA/EXPACS”, PLoS ONE 10(12): e0144679, 2015. The software including the developed model, EXPACS, is available online: http://phits.jaea.go.jp/expacs/

[32] S. Serre, S. Semikh, S. Uznanski, J.L. Autran, D. Munteanu, G. Gasiot, P. Roche, "Geant4 Analysis of n-Si Nuclear Reactions From Different Sources of Neutrons and Its Implication on Soft-Error Rate”, IEEE Trans. Nucl. Sci., Vol. 59, pp. 714-722, 2012.

[33] J. F. Ziegler, J. P. Biersack and U. Littmark, The Stopping and Range of Ions in Solids. New York: Pergamon Press, 1996.

[34] S. Martinie, T. Saad-Saoud, S. Moindjie, D. Munteanu, J.L. Autran, "Behavioral modeling of SRIM tables for numerical simulation", Nuclear Instruments and Methods in Physics Research Section B: Beam Interactions with Materials and Atoms, Vol. 322, pp. 2-6, 2014.

[35] H.H.K. Tang, "SEMM-2: A new generation of single-event-effect modeling tools," IBM Journal of Research and Development, Vol. 52, pp. 233-244, 2008. 
[36] S. Semikh, S. Serre, J. L. Autran, D. Munteanu, S. Sauze, E. Yakushev, S. Rozov, "The Plateau de Bure Neutron Monitor: Design, Operation and Monte Carlo Simulation”, IEEE Trans. Nucl. Sci., Vol. 59, pp. 303-313, 2012.

[37] Z. Torok, S.P. Platt, X.X. Cai, "SEE-inducing effects of cosmic rays at the HighAltitude Research Station Jungfraujoch compared to accelerated test data, ” RADECS 2007 Proc., pp.1-6, 2007.

[38] W. C. McColgin, C. Tivarus, C.C. Swanson, A.J. Filo, "Bright-Pixel Defects in Irradiated CCD Image Sensors”. MRS Proceedings, Vol. 994, pp. 0994-F12-06, 2007. 


\section{Figure Captions}

Figure 1. Schematics of direct/indirect ionization events resulting from the interaction of a single particle within the CCD structure and corresponding real images recorded with a CDD camera.

Figure 2. Views of the camera and of the experimental setup used in the work. The characteristics of the CCD imager are also summarized.

Figure 3. Examples of radiation-induced events captured by the CCD during exposures in complete darkness at sea level.

Figure 4. Experimental and simulated event rates (SPE+MPE) corresponding to the different experiment locations. The contributions of the different atmospheric particles and alpha particle emitters are also reported.

Figure 5. Experimental and simulated distributions of detected event sizes. The last point $(>10)$ represents the proportion of all events with a size greater than 10 pixels. 
Single particle interaction

DIRECT IONIZATION

Charged particle

(alpha, proton, muon)

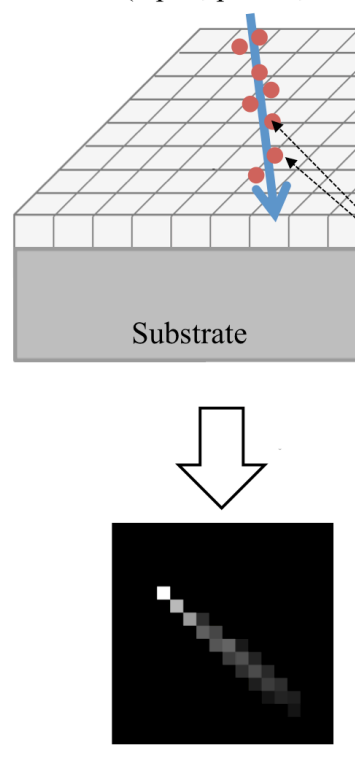

Single particle interaction INDIRECT IONIZATION

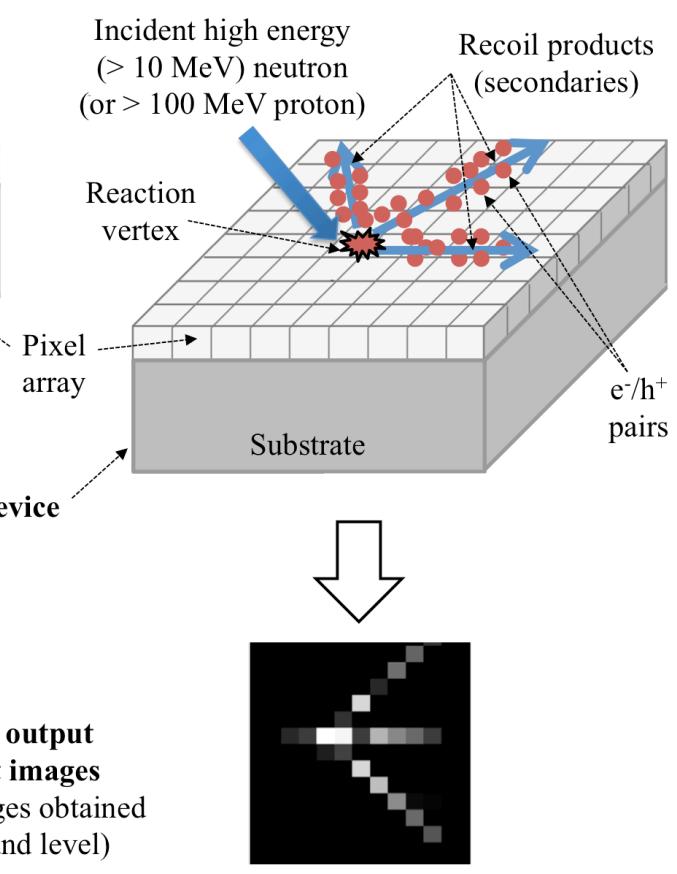

Figure 1. Saad Saoud et al. 


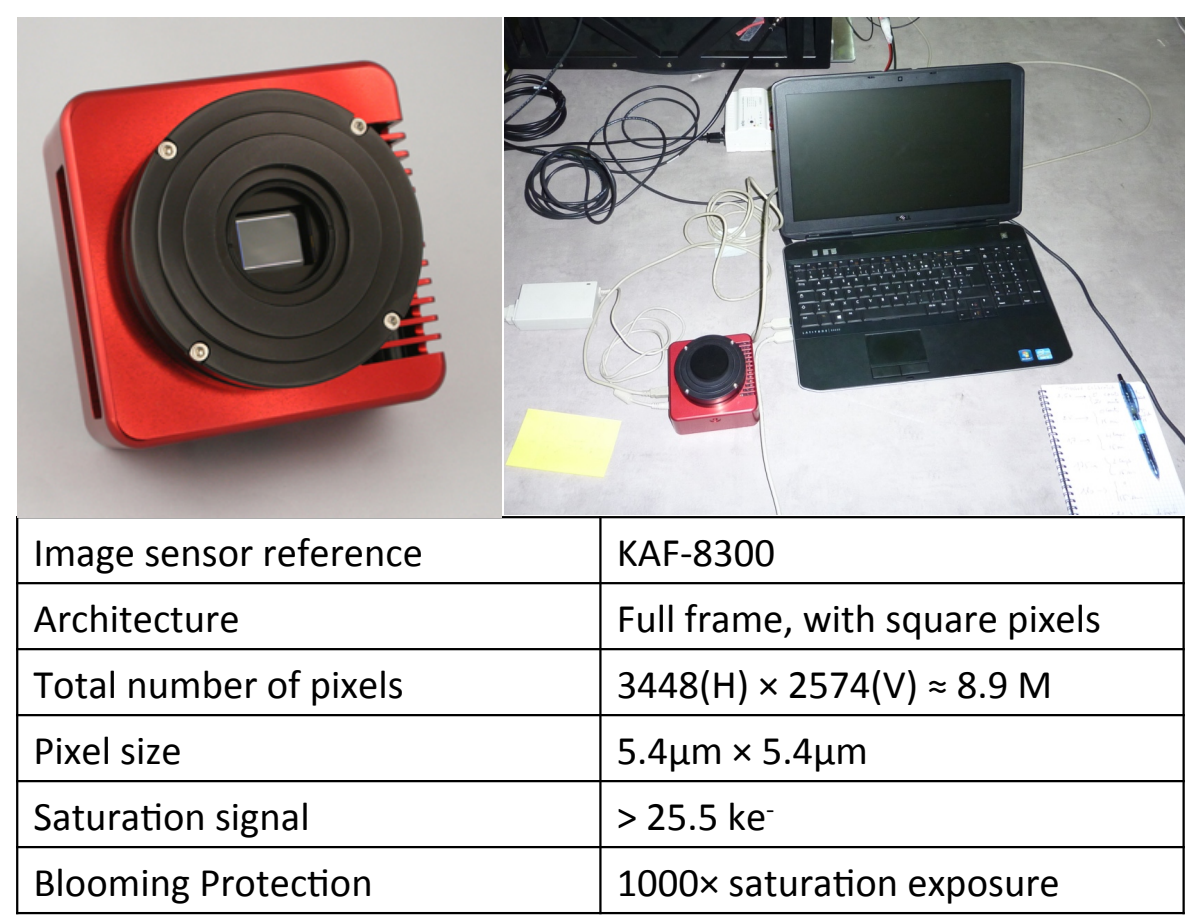

Figure 2. Saad Saoud et al. 

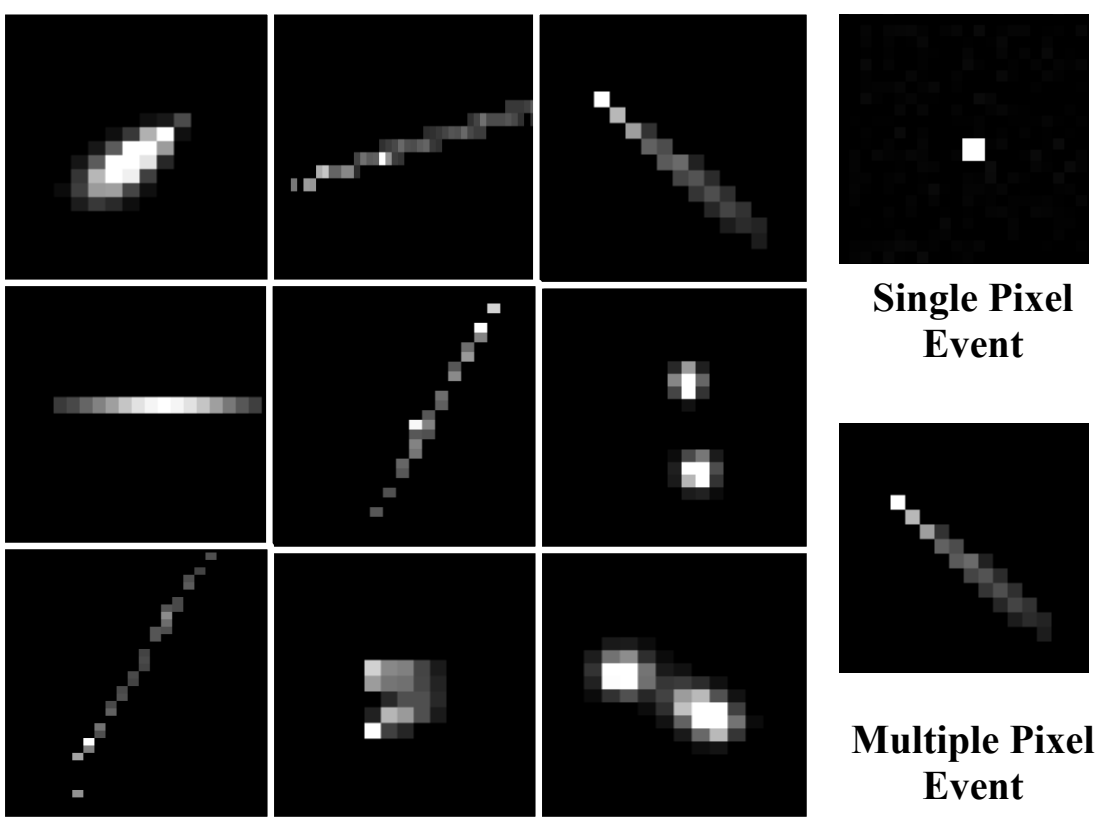

Single Pixel

Event

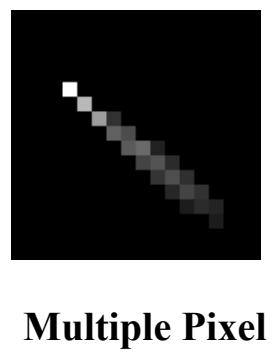

Event

Figure 3. Saad Saoud et al. 


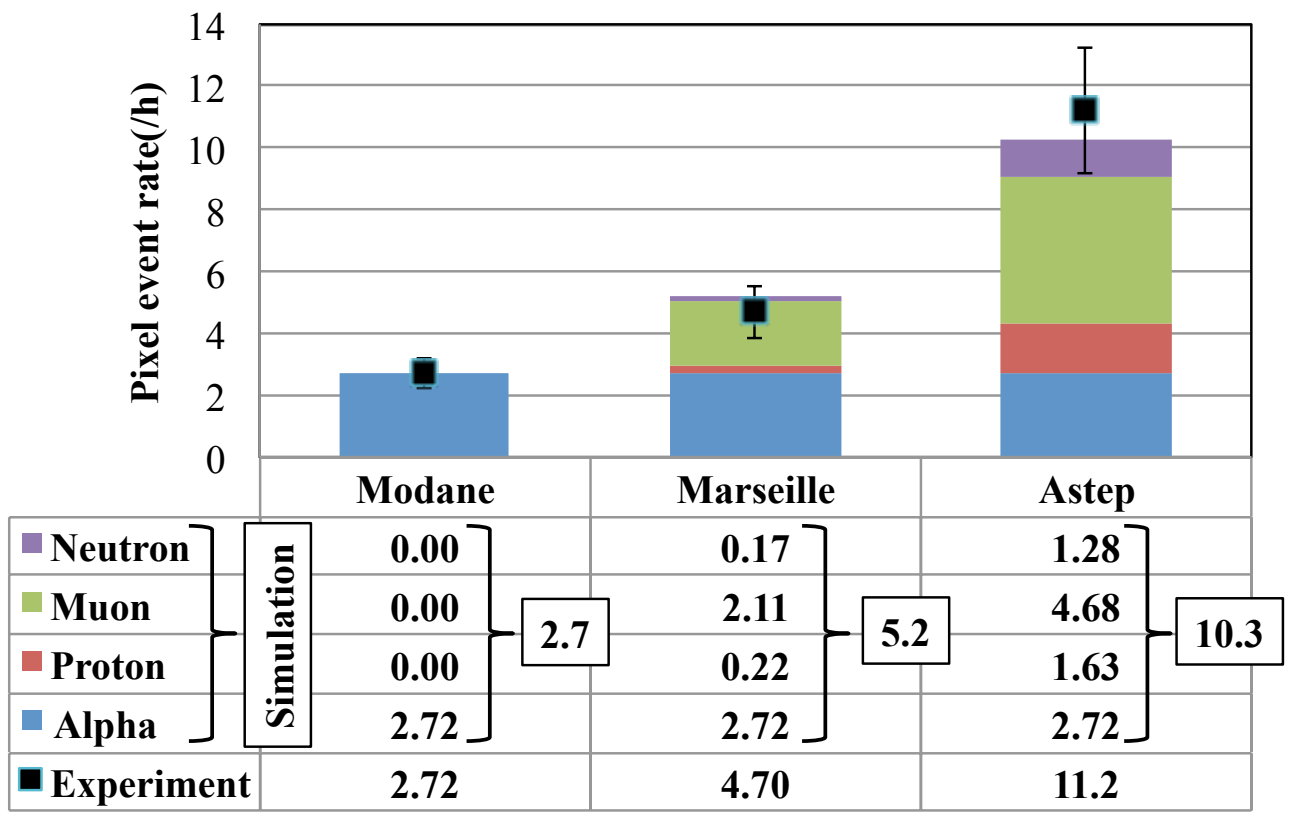

Figure 4. Saad Saoud et al. 


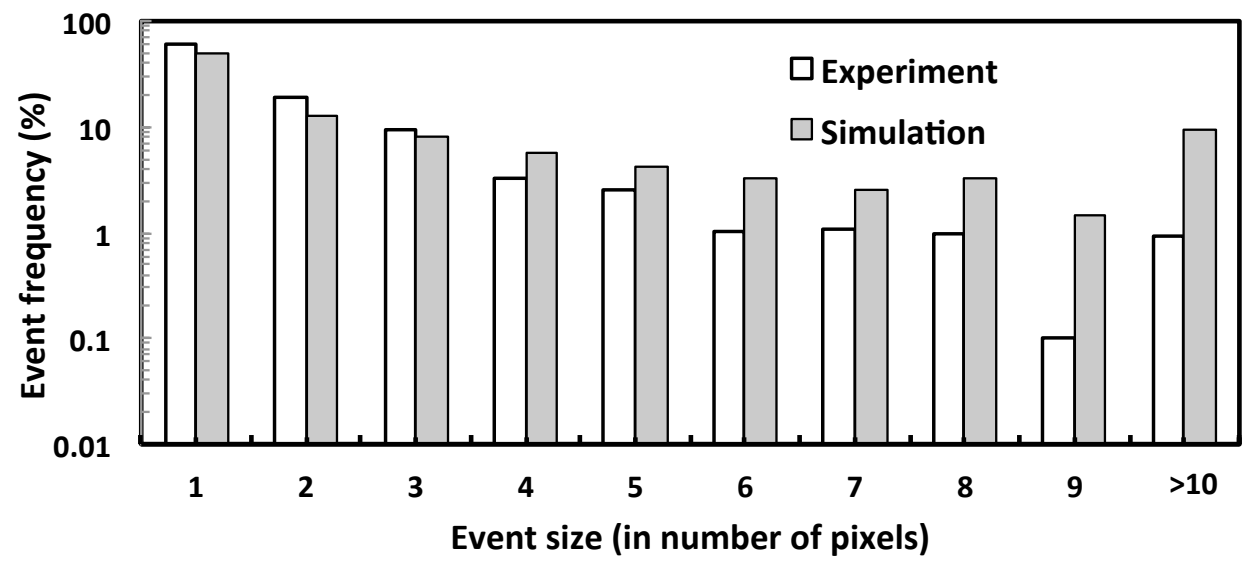

Figure 5. Saad Saoud et al. 